\title{
Cryptic Peripheral Ribosomal Domains Distributed Intermittently along Mammalian Myelinated Axons
}

\author{
Edward Koenig,, ${ }^{1}$ Rainer Martin, ${ }^{2}$ Margaret Titmus, ${ }^{1}$ and José R. Sotelo-Silveira ${ }^{3}$ \\ ${ }^{1}$ Department of Physiology and Biophysics, University at Buffalo School of Medicine, Buffalo, New York 14214, 2 Universität \\ Ulm, Sektion Elektronenmikroskopie, D-89081 Ulm, Germany, and 3BBiofísica, Instituto de Investigaciones Biológicas \\ Clemente Estable, Montevideo, Uruguay
}

\begin{abstract}
A growing body of metabolic and molecular evidence of an endogenous protein-synthesizing machinery in the mature axon is a challenge to the prevailing dogma that the latter is dependent exclusively on slow axoplasmic transport to maintain protein mass in a steady state. However, evidence for a systematic occurrence of ribosomes in mature vertebrate axons has been lacking until recently, when restricted ribosomal domains, called "periaxoplasmic plaques," were described in goldfish CNS myelinated axons. Comparable restricted RNA/ribosomal "plaque" domains now have been identified in myelinated axons of lumbar spinal nerve roots in rabbit and rat on the basis of RNase sensitivity of YOYO-1-binding fluorescence, immunofluorescence of ribosome-specific antibodies, and ribosome phosphorus mapping by electron spectroscopic imaging (ESI). The findings were derived from examination of the axoplasm isolated
\end{abstract}

The structural continuity of the extended axon depends on the steady-state maintenance of its protein mass. Goldscheider in the late nineteenth century was probably the first to postulate that the axon was maintained by "autochthonous metabolism" (see Barker, 1899). However, later in the twentieth century, axons were characterized ultrastructurally as lacking ribosomes (Palay and Palade, 1955; Peters et al., 1970). By default, axoplasmic transport (Grafstein and Foreman, 1980) appeared to afford the only means by which the axon compartment could be supplied with requisite proteins. The concept rapidly gave rise to a prevailing dogma. The principal tenets were that all axoplasmic proteins were synthesized in cognate cell bodies, that they were supplied to the axon via two slow transport rate groups, and that they were assumed to be metabolically stable during transport, irrespective of axon length (Lasek and Hoffman, 1976; Black and Lasek, 1980).

Prevailing views notwithstanding, some studies during this time indicated that mature axons may contain an endogenous proteinsynthesizing machinery (see Giuditta, 1980; Koenig, 1984). Moreover, it also became apparent that slowly transported proteins were not metabolically stable (Nixon, 1980; Nixon and Logvinenko, 1986 ) and that amino acid residues released during breakdown in the axon were reused locally (Nixon, 1980). A review of the current evidence for an endogenous machinery (Koenig and Giuditta, 1999; Alvarez et al., 2000) and a critique of slow transport theory (Alvarez et al., 2000) indicate that slow transport as a sole mechanism to explain maintenance and some aspects of the biology of long axons is not tenable.

In general, there have been only occasional reports of ribosomes in mature axons (Zelená, 1972; Martin et al., 1989; Pannese and

\footnotetext{
Received May 26, 2000; revised Aug. 25, 2000; accepted Aug. 28, 2000.

This work was supported by Grant IBN-9604841 from the National Science Foundation to E.K. We thank Dr. Joan A. Steitz for her generous supply of Y-10B antibodies.

Correspondence should be addressed to Dr. Edward Koenig, Department of Physiology and Biophysics, Cary Hall 321, University at Buffalo, Buffalo, NY 14214. E-mail: ekoenig@acsu.buffalo.edu.

Copyright (C) 2000 Society for Neuroscience $0270-6474 / 00 / 208390-11 \$ 15.00 / 0$
}

from myelinated fibers as axoplasmic whole mounts and delipidated spinal nerve roots. Ribosomal periaxoplasmic plaque domains in rabbit axons were typically narrow $(\sim 2 \mu \mathrm{m})$, elongated $(\sim 10 \mu \mathrm{m})$ sites that frequently were marked by a protruding structure. The domain complexity included an apparent ribosome-binding matrix. The small size, random distribution, and variable intermittent axial spacing of plaques around the periphery of axoplasm near the axon-myelin border are likely reasons why their systematic occurrence has remained undetected in ensheathed axons. The periodic but regular incidence of ribosomal domains provides a structural basis for previous metabolic evidence of protein synthesis in myelinated axons.

Key words: axoplasm; myelinated axons; ribosomes; RNA; YOYO-1; electron spectroscopic imaging; ESI; spinal nerves
Ledda, 1991; Sotelo et al., 1999). Recent experiments, however, performed on "axoplasmic whole mounts" isolated from myelinated fibers of goldfish CNS revealed a systematically organized distribution of restricted RNA-containing domains (Koenig and Martin, 1996). These restricted RNA domains were sites that often were identified in phase or DIC microscopy by a protruding structural correlate localized in the periphery of axoplasm of whole mounts and were called, therefore, "periaxoplasmic plaques." Electron spectroscopic imaging (ESI) of rRNA phosphorus confirmed that ribosomes were present in plaque domains and further indicated that polyribosomes probably corresponded to large fluorescent "puncta" in axoplasm after RNA staining by YOYO-1. ESI also revealed that ribosomes were attached to the inner zone of a matrix, comprising the overlying structural correlate of the domain.

The present report focuses on experiments conducted on axoplasmic whole mounts isolated from mammalian myelinated fibers in lumbar spinal nerve roots. Such preparations reveal restricted ribosome-containing domains that lie near the axolemma and have a random intermittent longitudinal distribution, similar to those referred to as periaxoplasmic plaques in myelinated axons of the goldfish CNS (Koenig and Martin, 1996). These domains are likely the focal centers of local translational activity that can account for protein synthesis in Mauthner and spinal root axons (Koenig, 1991) and may well be ubiquitous to myelinated axons as a class.

\section{MATERIALS AND METHODS}

Isolation of axoplasmic whole mounts from myelinated spinal root fibers. Lumbar spinal nerve roots that were used in the present study were dissected from dead rabbits or rats. The tissues were suspended in a modified gluconate-substituted calcium-free Cortland salt solution (Koenig and Martin, 1996) containing (in mM) $132 \mathrm{Na}$-gluconate, $5 \mathrm{KCl}, 20$ HEPES, 10 glucose, $3.5 \mathrm{MgSO}_{4}$, and 2 EGTA, pH 7.2, stored at $4^{\circ} \mathrm{C}$. Recovery of periaxoplasmic plaques on isolated axoplasmic whole mounts is best with fresh tissue and becomes increasingly variable and less likely from nerves stored for $>1 \mathrm{~d}$.

Compared with peripheral nerves, lumbar spinal nerve roots were nerves of choice in the present study because they are long, lack an epineurium, and have less interfasicular connective tissue that improves 
the efficiency of isolating axoplasm from multiple fibers simultaneously. A nerve root/rootlet, $3-5 \mathrm{~mm}$, was immersed in a solution of $30 \mathrm{~mm}$ zinc acetate, 0.1 M $N$-tris[hydroxymethyl]methylglycine (Tricine; Sigma, St. Louis, MO), and $0.1 \mathrm{M} N, N$-bis[hydroxyethyl]-2-aminoethane-sufonic acid

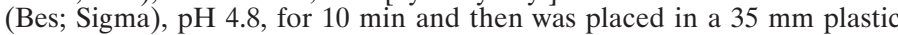
culture dish containing $2 \mathrm{ml}$ of a "pulling" solution, $\mathrm{pH}$ 5.5, and a "critical permissive concentration" (CPC) of aspartic acid, neutralized by arginine. The CPC usually was sharply defined for a given animal, in which the best recovery of ribosomal plaque domains varied within a limited range of concentrations of $35-45 \mathrm{~mm}$, but it could be as low as 30 or as much as 50 $\mathrm{mM}$ aspartate. The CPC was determined by making up test "pulling" solutions. The "pulling" solution contained an appropriate arginine aspartate concentration from a stock, $50 \mathrm{~mm}$ Bes, and $5 \mathrm{mM} \mathrm{Mg}$-acetate, $\mathrm{pH} 5.5$. The stock solution contained $0.2 \mathrm{M}$ aspartic acid (Sigma), 0.22 arginine (free base), $5 \mathrm{~mm} \mathrm{Na} \mathrm{N}_{3}$, and $0.1 \%$ Tween 20 (Bio-Rad, Hercules, CA) to reduce surface tension, $\mathrm{pH} 5.5$, and was stored at $4^{\circ} \mathrm{C}$. For each test, plaque occurrence was evaluated after staining with YOYO-1 (see below); two or three sprays were isolated at each of three concentrations that were separated by increments of $5 \mathrm{~mm}$ with respect to aspartate.

YOYO-1 staining of axoplasmic whole mounts. YOYO-1 iodide (491/509; Molecular Probes, Eugene, OR) was stored in DMSO as a 1:10 stock solution at $-15^{\circ} \mathrm{C}$. After whole-mount sprays were attached to a coverslip, $1 \mu \mathrm{l}$ of stock YOYO- 1 was added to the pulling medium (final concentration, 1:5000) for $15 \mathrm{~min}$. The YOYO-1 was washed out by brief immersion in acidified $0.15 \mathrm{M}$ ammonium acetate (i.e., $\mathrm{NH}_{4} \mathrm{OAc}$; $\mathrm{pH}$-adjusted to 4.5 with acetic acid) and $0.1 \%$ Tween 20 . For fluorescence microscopy the coverslip with axoplasmic sprays was mounted on a flow-thru chamber. The chamber was constructed by inverting the coverslip over spacers $(0.5-1 \mathrm{~mm}$ thick) made of Silastic elastomer (Dow Corning, Midland, MI) attached to a large glass coverslip $(35 \times 50 \mathrm{~mm})$ taped to a thin "U"-shaped metal plate, and the well was filled with acidified $\mathrm{NH}_{4} \mathrm{OAc}$ solution.

Immunofluorescence staining. Axoplasmic whole mounts attached to a coverslip were fixed by immersion in $3.75 \%$ paraformaldehyde in $0.1 \mathrm{M}$ sodium diethylmalonate $[0.1 \mathrm{~m}$ diethylmalonic acid (Aldrich, Milwaukee, WI), pH-adjusted to 7.2 with $\mathrm{NaOH}$ ] and $0.1 \%$ Tween $20, \mathrm{pH} 7.2$, for 15 min. They were washed in $0.15 \mathrm{M}$ ammonium acetate and $0.1 \%$ Tween 20 , $\mathrm{pH}$ 6.7, three times for $5 \mathrm{~min}$ each and then immersed in an immunoblocking solution, composed of $25 \mathrm{~mm}$ Tris $\mathrm{HCl}, 0.9 \% \mathrm{NaCl}, 3.75 \%$ glycine, $1 \%$ of normal goat and/or donkey serum, $0.05 \%$ Tween 20 , and $5 \mathrm{mM} \mathrm{NaN}_{3}$ for $15 \mathrm{~min}$. Incubation with primary antibody was for $1 \mathrm{hr}$ on a rocker. Coverslips were washed three times with a working buffer (i.e., blocking buffer with $0.1 \%$ serum) and incubated for $45 \mathrm{~min}$ with a secondary antibody conjugated to one of two Alexa fluorophores (Molecular Probes) having an excitation maximum at either 488 or $546 \mathrm{~nm}$. The immunostained specimens were washed further three times for 5 min each before being mounted over spacers of the flow-thru chamber for microscopic examination (see above).

The immunoreagents that were used were monoclonal antibody $(\mathrm{mAb})$ Y-10B (a generous gift of Dr. Joan A. Steitz, Yale University, New Haven, $\mathrm{CT}$ ) and human autoantibodies against ribosomal $\mathrm{P}$ antigen, purchased from ImmunoVision (Springdale, AR). Y-10B is a monoclonal antibody specific for the large ribosomal subunit RNA (Lerner et al., 1981), and human ribosomal $\mathrm{P}$ antigen autoantibodies react with a complex of three specific proteins associated with the large ribosomal subunit (Chu et al., 1991). Ribonuclease (RNase) digestion of axoplasmic whole mounts (see below) completely eliminated immunoreactivity of Y-10B (see below). Primary antibodies were used at 1:200 in a working buffer of the same composition.

Ribonuclease digestion of axoplasmic whole-mount sprays. Two sets of axoplasmic whole-mount sprays attached to coverslips, in which the presence of periaxoplasmic plaques was confirmed by YOYO-1 staining, were fixed with $3.75 \%$ paraformaldehyde and $0.1 \mathrm{~m}$ diethylmalonic acid, $\mathrm{pH}$ adjusted to 7.2 with $\mathrm{NaOH}$. One spray set was incubated with $0.4 \mathrm{mg}$ of ribonuclease (RNase) $/ \mathrm{ml}$ (Worthington Biochemical, Freehold, NJ) in $0.15 \mathrm{M}$ $\mathrm{NH}_{4} \mathrm{OAc}$ and $0.1 \%$ Tween $20, \mathrm{pH} 6.8$, at $37^{\circ} \mathrm{C}$ for $45 \mathrm{~min}$, and the second was incubated with buffer alone. Axoplasmic whole mounts then were stained again with YOYO-1 or processed for mAb Y-10B immunofluorescence to evaluate periaxoplasmic plaque occurrence.

Microscopy. For routine epifluorescence, DIC, or phase-contrast microscopy the specimens were examined with an Olympus BHS microscope with $25 \times$ (numerical aperture, NA, 0.60), 40× (NA, 0.70), and 100× oil immersion (NA, 1.25) objectives. Computer-assisted (Power Macintosh G3) gray scale video images were acquired with an air-cooled CCD MTI camera (model 3001-RC) mounted on the Olympus microscope, using a Scion LG-3 framegrabber (Scion, Frederick, MD) and a Dage DSP-2000 image processor (Dage-MTI, Michigan City, IN).

Image analysis of periaxoplasmic plaques. Fluorescent images of wholemount sprays after staining by YOYO-1 or immunostaining by Y-10B were captured at $40 \times$ power, and single whole-mount segments of variable lengths in the plane of focus were selected for image processing and analysis. Acquired images were analyzed with IPLab (Scanalytics, Fairfax, VA) software. To analyze in-focus images of plaques in some cases, we captured the image of the same whole-mount segment in more than one focal plane. A single out-of-focus plaque image acquired in one focal plane was cut and replaced by pasting in the corresponding in-focus image from the second focal plane. Analysis of fluorescent plaques was highlighted and selected by thresholding. This also eliminated from analysis the weaker punctate staining of mitochondria in axoplasm. The following geometrical variables were analyzed: plaque length, plaque width, plaque area, axial interplaque distance, axoplasmic whole-mount diameter, and the length of the whole-mount segment from which plaques had been selected for analysis.

Whole-mount bundle and delipidated nerve root preparations for ESI. Use of heavy metals is precluded for energy loss elemental mapping by ESI. Therefore, it was necessary to use either bundles of isolated axoplasmic whole mounts or lipid-extracted nerve specimens. Axoplasmic wholemount sprays were isolated from a ventral nerve rootlet. Each spray was condensed into a compact bundle by briefly drawing the spray out of solution except for one end, resubmerging it, and attaching the condensed bundle at both ends to a coated coverslip (see above). Several whole-mount bundles attached to a coverslip in this manner were fixed by immersion in $2.5 \%$ glutaraldehyde in $0.1 \mathrm{~m}$ sodium diethylmalonate, $\mathrm{pH} 7.2$, for $1 \mathrm{hr}$, dehydrated by an ethanol series, and embedded in Epon 812

In the delipidation procedure a segment of ventral nerve root was fixed by immersion in $2.5 \%$ glutaraldehyde and $0.1 \mathrm{M}$ Na-diethylmalonate, $\mathrm{pH}$ 7.2 , for $3 \mathrm{hr}$. Then the nerve was removed, blotted lightly, and immersed in chloroform-methanol $(2: 1, \mathrm{v} / \mathrm{v})$ for $15 \mathrm{~min}$; the organic solvent mixture was replaced by absolute methanol for $10 \mathrm{~min}$. The nerve was rehydrated in $0.15 \mathrm{M}$ ammonium acetate, $\mathrm{pH} 6.8$, dehydrated through an ethanol series, equilibrated in propylene oxide, and embedded in an Epon 812 mixture on a coverslip.

The glass coverslip was removed from the embedded specimen by immersion in $49 \%$ hydrofluoric acid in an ice bath, and the plastic wafer was washed in tap water $(20 \mathrm{~min})$. The whole-mount bundle or nerve specimen was cut out, and acceptable portions were divided into $\sim 1 \mathrm{~mm}$ segments and mounted on individual blocks for sectioning. Ultrathin sections $(10-20 \mathrm{~nm})$ were cut from selected blocks. They were collected on uncoated 700-mesh grids and examined in a Zeiss CEM 902 transmission microscope equipped with an integrated electron energy spectrometer (Zeiss, Oberkochen, Germany) and an image analysis system from Kontron (Munich, Germany).

\section{RESULTS}

Axoplasmic whole mounts were isolated from both dorsal and ventral nerve root fibers of the rabbit and rat to evaluate plaque occurrence, morphology, and distribution. Because gross morphological features and distribution of periaxoplasmic plaques appeared qualitatively similar in both dorsal and ventral nerve fibers, and in the two species, most of the findings reported below are based on the generally larger rabbit axons of ventral root fibers unless otherwise noted.

\section{Conditions for isolating axoplasmic whole-mount preparations with periaxoplasmic plaques}

Native axoplasm behaves as a viscoelastic solid, in which the tensile strength that is required for isolation depends on axon diameter and the content of neurofilaments (Gilbert et al., 1975; E. Koenig, unpublished observations). Although periaxoplasmic plaques initially were discovered in native axoplasm of a whole mount translated from the large goldfish myelinated Mauthner fiber (Koenig and Martin, 1996), there was a significant likelihood that the cortical layer of the whole mount would be disrupted during translation. Axoplasm of smaller axons, such as those isolated from myelinated fibers of mammalian cranial (Koenig, 1965) or lumbar spinal nerve roots (Koenig, 1991), requires increased tensile strength to withstand the axial stress and frictional forces that are generated during translation. Zinc denaturation increases tensile strength, and such treatment increases the efficiency of isolation by allowing multiple axoplasmic whole mounts to be removed and then attached to a coverslip as a "spray" under low magnification (e.g., 12×; see Materials and Methods). Whole mounts can be viewed in a dark microscopic field by light scattering, using a horizontally oriented halogen light source (Fig. 1).

Although denatured axoplasm is easy to isolate by translation, conditions that favor the recovery of plaques cannot be defined precisely. In the goldfish Mauthner axon, plaque formations are associated closely with or are actual inclusions of the cortical F-actin layer (Koenig and Martin, 1996). This is a thin cytoskeletal layer subjacent to the plasma membrane that surrounds an axoplasmic core, made up mainly of axially oriented cross-bridged neurofilaments and microtubules (Hirokawa, 1991). The translation technique produces an abrupt shearing of interactions that normally cross-link the F-actin layer to the membrane and potential transmembrane elements in the intact myelinated fiber. As axo- 
Axoplasmic wholemount "sprays"

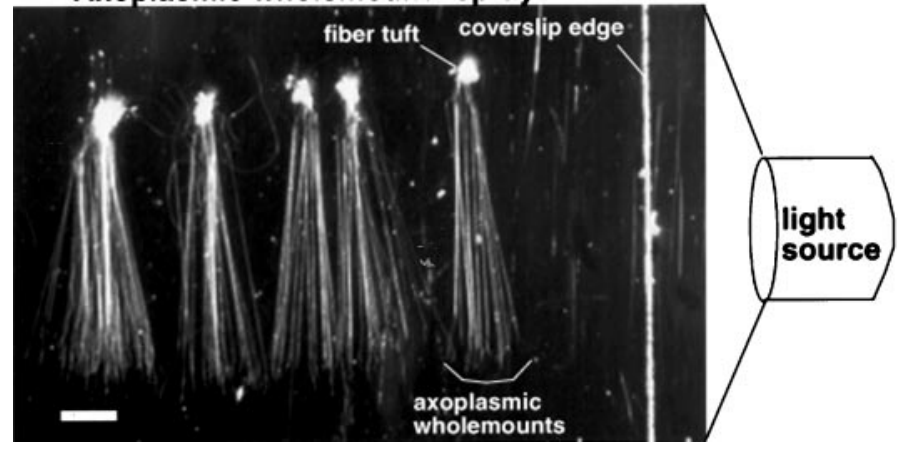

Figure 1. A dark-field view through a dissecting microscope of five axon sprays isolated from rabbit ventral nerve root fibers that were attached to a coverslip surface. A spray is defined as multiple isolated axoplasmic whole mounts originating in a nerve fiber tuft remnant that was used to grasp the spray. Scale bar, $1 \mathrm{~mm}$.

plasm slides along the lumen formed by the myelin sheath, there is further shearing by frictional forces, depending on stiffness and compression of the sheath. Although the present protocol (see Materials and Methods) promotes the recovery of plaques, the $\mathrm{CPC}$, the incidence of whole mounts with plaques, the frequency of plaque occurrence on individual whole mounts, and the structural integrity of plaques that have been recovered (see below) are all affected by biological variation and the amount of time that tissue is stored at $4^{\circ} \mathrm{C}$.

\section{RNA and ribosomes in periaxoplasmic plaque domains located on axoplasmic whole mounts isolated from ventral root fibers}

When axoplasmic whole mounts are isolated under permissive conditions (i.e., at the $\mathrm{CPC}$ of aspartate), periaxoplasmic plaques become visible at the surface of whole mounts after fluorescence staining with a high-affinity nucleic acid-binding dye such as YOYO-1. Typically, plaques are sharply defined, elongated fluorescent domains (Fig. 2) in which background fluorescence in axoplasm is low, except for weak punctate fluorescence because of staining of mitochondria (see Fig. 4D). Uptake of dye by mitochondria and fluorescence intensity are variable, depending on incubation time and treatments that affect mitochondrial permeability. Spatially discrete plaque domains are restricted to the surface boundary of the whole mount and are readily distinguishable from the weaker, punctate fluorescence of mitochondria dispersed throughout the volume of axoplasm (see also Koenig and Martin, 1996).

At a concentration of $5-10 \mathrm{~mm}$ above or below the CPC of aspartate, stained plaques may be absent entirely, or there may be only a few whole mounts with scattered plaques that appear "skeletonized," in which only fluorescent remnants are apparent. At concentrations moderately above the CPC, whole-mount axoplasm also may exhibit an overall diff use, nonspecific bright background fluorescence. Because sprays isolated over a range of aspartate concentrations that includes the CPC are stained at the same time by YOYO-1, the abrupt nonspecific increase in background fluorescence of sprays isolated above the CPC would appear to reflect an indeterminate change in properties of axoplasm.

Figure 2 shows typical examples of whole-mount sprays isolated from rabbit and rat ventral root fibers with plaques in acquired images. Plaques appear randomly distributed at intermittent intervals along the whole mount, and staining is eliminated by incubation with RNase (data not shown; see below). Their dimensions as well as interplaque spacing vary considerably (see below). The morphological features and distributional patterns characteristic of whole mounts isolated from myelinated ventral root fibers are also typical of whole mounts isolated from dorsal root fibers (data not shown) and similar to whole mounts isolated from ordinary myelinated fibers of the goldfish spinal cord (Koenig and Martin,
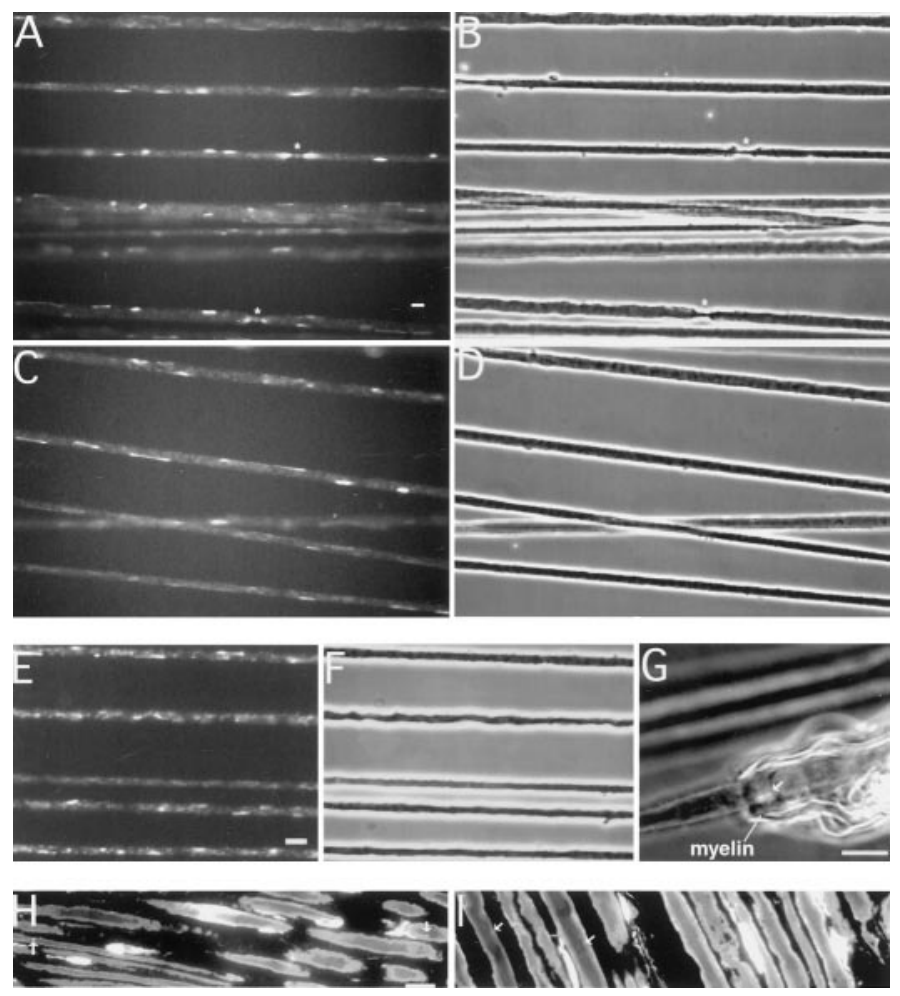

Figure 2. Periaxoplasmic plaques distributed along axoplasmic whole mounts isolated from rabbit and rat ventral root nerve fibers. $A, C$, Lowpower micrographs of randomly selected portions of rabbit whole-mount sprays in which periaxoplasmic plaque domains are revealed by fluorescence staining with YOYO-1. Note that plaques are distributed around the surface of the whole mount and may appear indistinct because they are out of the plane of focus, either on the same whole mount or on another whole mount. $B, D$, Phase-contrast images corresponding to those shown in $A$ and $C$. Nodes of Ranvier are indicated by an asterisk. $E, F$, Plaque domains associated with axoplasmic whole mounts on a portion of a spray isolated from rat ventral root fibers (note higher magnification). $G$, An isolated rat axoplasmic whole mount with a partially myelinated segment in which a plaque (arrow) that was stained fluorescently by YOYO-1 is shown near the border of the ensheathed portion (the image was acquired by simultaneous phase and epifluorescence microscopy). $H, I$, Representative examples of $0.5 \mu \mathrm{m}$ sections of Epon-embedded rabbit ventral root fibers stained with YOYO-1 in which putative plaques are identified (arrows). Scale bars: $A-G$, $10 \mu \mathrm{m} ; H, 15 \mu \mathrm{m}$.
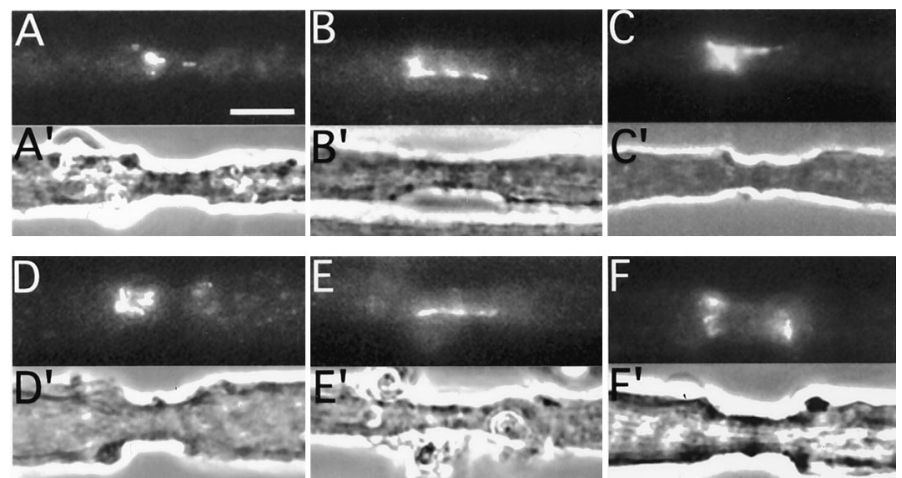

Figure 3. Examples of plaque-like YOYO-1 fluorescence in nodal/paranodal regions of axoplasmic whole mounts isolated from rabbit ventral root fibers. $A-F$, Fluorescence images show variations in RNA fluorescence patterns within nodal and paranodal regions. $A^{\prime}-F^{\prime}$, Corresponding phase images. Scale bar, $10 \mu \mathrm{m}$.

1996). Although most periaxoplasmic plaques are located along internodes, fluorescent plaque-like domains also are seen occasionally within nodal and/or paranodal regions (Fig. 3). Occasionally, an isolated whole mount may have a segment to which myelin may still adhere. Axoplasm within the ensheathed portion usually does 

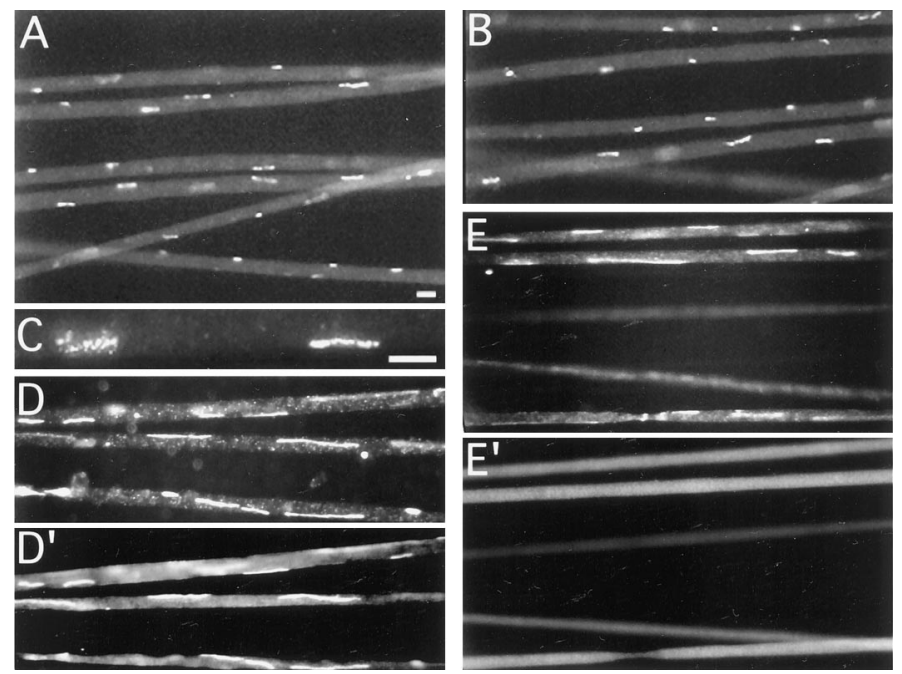

Figure 4. Immunofluorescence staining of ribosomes in periaxoplasmic plaques by monoclonal antibody Y-10B distributed along axoplasmic whole mounts isolated from rabbit ventral root nerve fibers and RNase sensitivity. $A, B$, Randomly selected portions of rabbit whole-mount sprays in which periaxoplasmic plaque domains are revealed at low magnification by immunofluorescence staining with $\mathrm{Y}-10 \mathrm{~B}$, a monoclonal antibody $(\mathrm{mAb})$ that binds to the large ribosome subunit RNA (Lerner et al., 1981). C, Highresolution images of periaxoplasmic plaques reveal the discrete, large, punctate character of immunofluorescence staining by Y-10B. $D$, A region of a spray from one spray set showing fluorescence staining of plaques by YOYO-1 $\left(\lambda_{\max }, 509 \mathrm{~nm}\right)$. Note that the weak punctate fluorescence after YOYO staining is associated with mitochondria within axoplasm. $D^{\prime}$, The corresponding region imaged in $D$ after fixation and immunofluorescence staining by $\mathrm{mAb} \mathrm{Y}-10 \mathrm{~B}\left(\lambda_{\max }, 575 \mathrm{~nm}\right)$ showing a direct correspondence in plaque staining between YOYO-1 and mAb Y-10B. Out-of-focus fluorescence originates from plaques located on opposite surfaces. $E$, A region of a spray from a second spray set showing fluorescence staining of plaques by YOYO-1. $E^{\prime}$, The corresponding region imaged in $E$ after fixation, RNase digestion, and immunofluorescence cytochemistry showing the absence of plaque immunofluorescence staining by mAb Y-10B. YOYO-1 did not restain plaques after RNase (data not shown). Scale bars: $A-E^{\prime}, 10 \mu \mathrm{m}$.

not stain during a short incubation with YOYO-1; however, a plaque domain may become stained if it is located near the border of the myelinated segment, as illustrated in Figure $2 G$ (arrow). Examples such as the latter provide confirmation of the occurrence of periaxoplasmic plaques in the myelin-ensheathed state.

Finally, our experience in testing standard histological sections as an optional means for studying periaxoplasmic plaques leads us to conclude that a conventional approach offers little merit. Representative examples of $0.5 \mu \mathrm{m}$ sections of Epon-embedded rabbit ventral root fibers stained with YOYO-1 are shown in Figure 2, $H$ and $I$, in which a few putative plaques are identified (arrows). The principal problem with this approach is the ambiguity inherent in identifying very small elongated domains, distributed randomly at the periphery of the axon (see below), in which domain exposure depends on the plane of section and usually is truncated to a variable extent. In addition, the bright fluorescence of nuclei and the RNA-rich cytoplasm of adjacent Schwann cells can obscure the comparatively weak fluorescence signal originating from a small discrete source such as a plaque domain located close to the axon-myelin interface. At present, therefore, isolation of axoplasmic whole mounts, notwithstanding the limitations of the technique from the standpoints of recovery and structural preservation, still appears to provide the best mode of studying periaxoplasmic plaques in myelinated axons.

YOYO-1 is a high-affinity dye that binds to RNA and DNA, and its nonspecific binding properties provide no information about ribosomes. Monoclonal antibody Y-10B, however, binds to the large ribosomal subunit RNA (Lerner et al., 1981) and was used to probe for the occurrence of ribosomes in periaxoplasmic plaque domains by immunofluorescence microscopy. Immunofluorescence staining yielded stereotypical plaque domains (Fig. 4). Although the fluorescence staining at low magnification appeared diffuse, it was consistently punctate at higher optical resolution (Fig. 4C). Human autoantibodies against ribosomal $\mathrm{P}$ antigen also produced similar punctate immunofluorescence staining of plaque domains (data not shown). In the Mauthner axon the larger fluorescent puncta, which spatially define the plaque domain, correlated with polyribosomal clusters at the electron microscopic level (Koenig and Martin, 1996). Mitochondria were not stained by the mAb Y-10B.

Inasmuch as mAb Y-10B immunoreacts with the RNA of the large ribosomal subunit (Lerner et al., 1981), immunoreactivity should be sensitive to RNase. To test mAb Y-10B immunospecificity, we prepared two sets of sprays on separate coverslips and identified plaques by YOYO-1 fluorescence staining (Fig. 4D,E). One set of sprays was incubated with RNase, and the second one was incubated with buffer alone (see Materials and Methods). Then the two sets of sprays were processed for mAb Y-10B immunocytochemistry. The results showed that (1) immunofluorescence staining of plaques by $\mathrm{mAb} \mathrm{Y}-10 \mathrm{~B}$ corresponded to fluorescence staining of the same plaque domains by YOYO-1 (Fig. $4 D, D^{\prime}$ ), and (2) immunofluorescence staining of plaques previously identified by YOYO-1 fluorescence was eliminated completely after RNase digestion (Fig. $4 E, E^{\prime}$ ) and was not restained by YOYO-1 (data not shown). The findings indicate, therefore, that plaque domains contain RNA and that the discrete punctate immunoreactivity of $\mathrm{mAb} Y-10 \mathrm{~B}$ is probably attributable to rRNA. The identification of ribosomes in plaque domains by $\mathrm{mAb} \mathrm{Y}-10 \mathrm{~B}$ and by anti-human ribosomal $\mathrm{P}$ antigen (data not shown) is consistent with ESI mapping of rRNA phosphorus at an electron microscopic level (see below).

\section{Phase structural correlates of periaxoplasmic plaque domains}

Well preserved periaxoplasmic plaques, identified by fluorescence staining on whole mounts, frequently are marked by structural correlates protruding at the surface of the whole mount (Fig. $5 A, A 1)$. At low magnification the entire plaque domain, including the phase correlate, is fluorescent. As revealed by mAb Y-10B immunofluorescence at higher optical resolution, however, there is a distinction between the structural correlate and fluorescent putative polyribosomal puncta, as seen by comparing corresponding phase and fluorescence images (Fig. 5). The configuration of structural correlates generally appears to correspond to the distribution of underlying fluorescent "puncta," but the overlying structure usually extends beyond the boundary of the puncta distribution. Structural correlates of plaque domains in the goldfish Mauthner axon are made up of a nondescript matrix to which ribosomes are attached at the inner zone (Koenig and Martin, 1996), and the possibility of matrix-ribosome interactions in mammalian plaques also is suggested by findings at the electron microscopic level (see below).

Preservation of structural correlates during isolation of axoplasmic whole mounts shows biological variability in that the latter are more resistant to disruption in some animals than in others. Thus, they may appear intact, as remnants (i.e., "skeletonized") because of disruption, or they may be absent entirely because they were "excavated" during translation. With some exceptions, the frequency of recovery of structural correlates usually diminishes with the storage of tissue at $4^{\circ} \mathrm{C}$.

\section{Morphological and distributional features of periaxoplasmic plaques in whole mounts from rabbit ventral nerve root fibers}

Axoplasmic whole-mount sprays were isolated from rabbit ventral nerve root fibers in which periaxoplasmic plaques were visualized by either YOYO-1 fluorescence staining or by $\mathrm{mAb}$ Y-10B immunofluorescence staining. The sprays were deemed to have normal plaque abundance and distribution. A limited series of in-focus images of single whole-mount segments, each ranging from 200 to $250 \mu \mathrm{m}$ in length, was selected from a set of acquired images of 


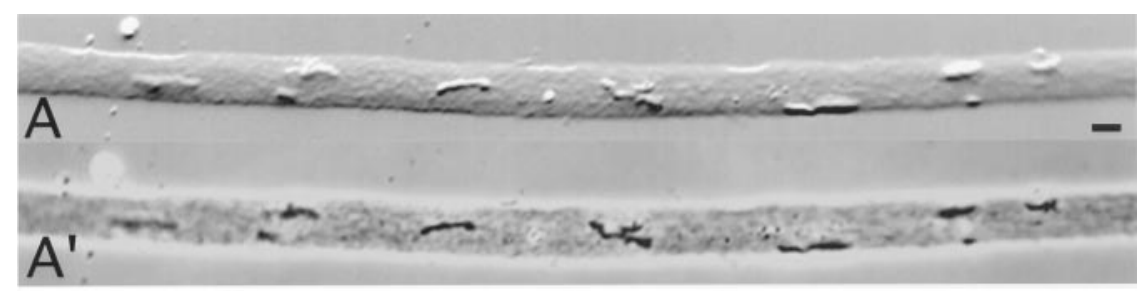

Figure 5. Structural correlates of periaxoplasmic plaques and a comparison of their shapes with distributions of fluorescent putative polyribosomal puncta immunostained by mAb Y-10B. $A$, Differential interference contrast (DIC) and corresponding phasecontrast images $\left(A^{\prime}\right)$ of plaque structural correlates protruding at the surface of an axoplasmic whole mount. $B-H$, Phase-contrast images of overlying plaque structural correlates and corresponding mAb Y-10B immunofluorescence images $\left(B^{\prime}-H^{\prime}\right)$ of underlying putative polyribosomal "puncta." $B^{\prime \prime}-H^{\prime \prime}$, Phase $(B-H)$ and corresponding fluorescence images $\left(B^{\prime}-H^{\prime}\right)$ were positioned in registration, and transparencies of the superimposed images were adjusted to reveal both structural and fluorescence distributions. Most plaque domains show a close correspondence between the shape of structural correlates and underlying fluorescent puncta distributions. Scale bars: $A-H^{\prime \prime}, 10 \mu \mathrm{m}$.

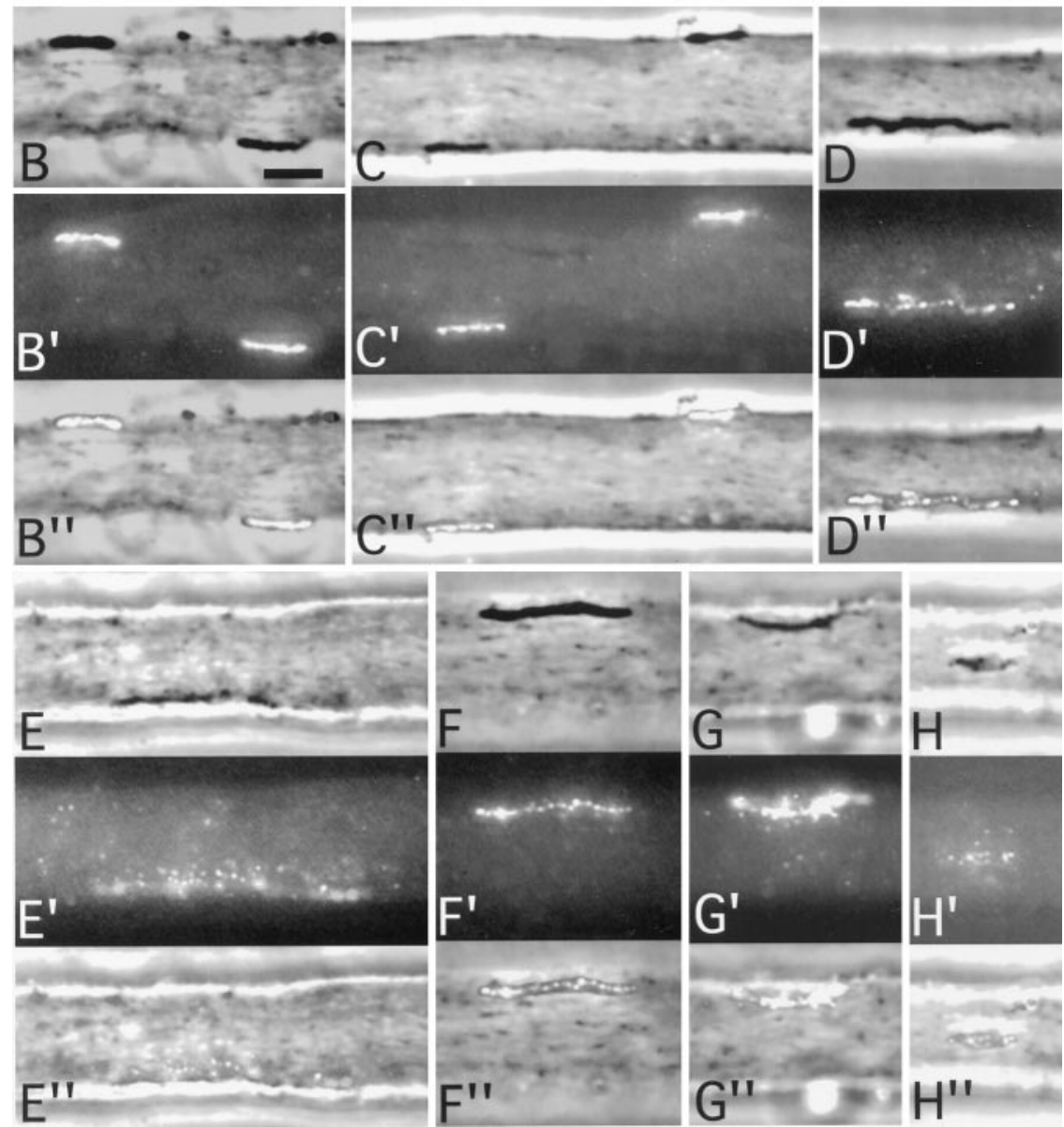

whole-mount sprays, and several size and distributional variables of periaxoplasmic plaques were measured (see Materials and Methods). From 3 to 22 plaques per segment were analyzed in 42 whole-mount segments after staining with YOYO-1 and in 10 whole-mount segments after immunostaining with mAb Y10-B. Most of the whole mounts that were examined ranged between 4 and $12 \mu \mathrm{m}$ in diameter (Fig. 6A).

Table 1 contains a summary of size characteristics of periaxoplasmic plaques as determined by image analysis of the larger YOYO-1 fluorescence series and the smaller mAb Y-10B immunofluorescence series. Although there was an apparent difference in the mean plaque length between the two groups, the difference

Table 1. Size characteristics of periaxoplasmic plaques in axoplasmic whole amounts isolated from rabbit ventral root fiber

\begin{tabular}{llll}
$\begin{array}{l}\text { Mode of } \\
\text { staining }\end{array}$ & $\begin{array}{l}\text { Overall average } \\
\text { plaque length } \\
(\text { mean } \pm \mathrm{SD})\end{array}$ & $\begin{array}{l}\text { Average plaque } \\
\text { width/segment } \\
(\text { mean } \pm \mathrm{SD})\end{array}$ & $\begin{array}{l}\text { Overall average } \\
\text { plaque area } \\
(\text { mean } \pm \mathrm{SD})\end{array}$ \\
\hline YOYO-1 & $9.9 \pm 5.9 \mu \mathrm{m}$ & $2.1 \pm 0.7 \mu \mathrm{m}$ & $13.1 \pm 11.3 \mu \mathrm{m}^{2}$ \\
$n$ & 305 & 39 & 508 \\
Y-10B & $8.5 \pm 5.7 \mu \mathrm{m}$ & $2.1 \pm 0.3 \mu \mathrm{m}$ & $13.0 \pm 8.1 \mu \mathrm{m}^{2}$ \\
$n$ & 73 & 10 & 73 \\
\hline
\end{tabular}

did not reach statistical significance ( $p<0.07$; two-tailed Student's $t$ test). The average plaque width, which is based on the average of the means of plaque widths per segment in each series, and the average plaque area for all measured plaques were quite similar between the groups.

The data sets from the YOYO-1 and mAb Y-10B series were pooled, and several parameters were evaluated graphically in Figure 6 . Although plaque length averaged $\sim 10 \mu \mathrm{m}$ (see Table 1 ), the distribution of plaque lengths in the population was asymmetric (Fig. 6B), with lengths varying considerably within individual whole-mount segments (Fig. 6C). Similarly, the distribution of plaque areas was skewed also (Fig. $6 F$ ), and areas varied as well within whole-mount segments (Fig. $6 E$ ). Finally, the average axial interplaque distance per segment $(n=50)$ was $23.0 \pm 12.3 \mu \mathrm{m}$; the overall distribution is shown in Figure $6 D$.

Summation of individual plaque areas per whole-mount segment yielded a mean $( \pm \mathrm{SD})$ that composed $2.4 \pm 1.4 \%$ of the surface area of the segment. However, the mean total plaque area per whole-mount segment correlated neither with the nominal surface areas of whole-mount segments nor with the nominal volumes of whole-mount segments. Potential systematic differences in the size and/or distributional characteristics of periaxoplasmic plaques that were based on age or weight of the animals were not investigated. The possibility that there could be differences, as in plaque lengthsfor example, can be inferred from a comparison of the spray 

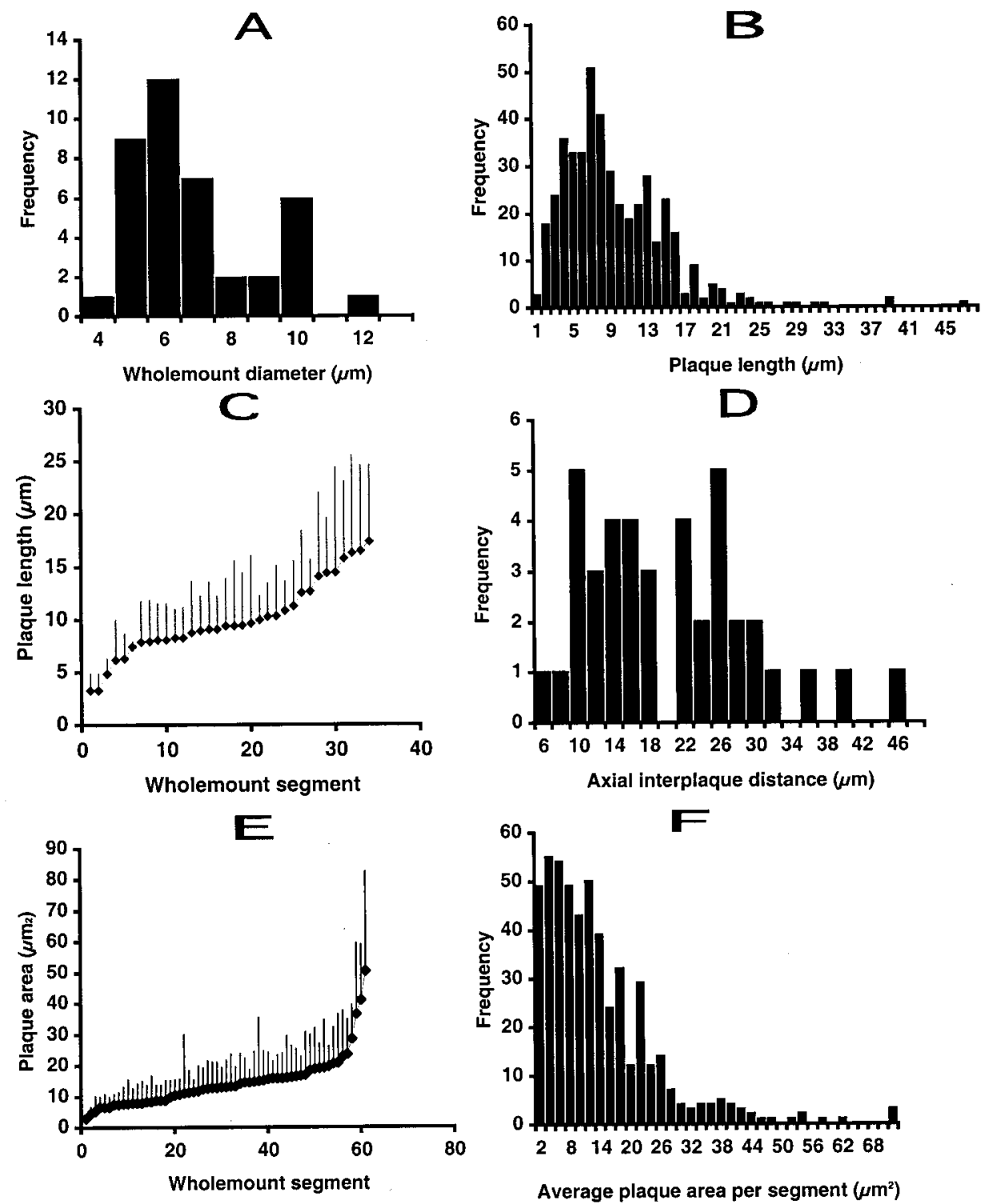

Figure 6. Analysis of axoplasmic whole mounts isolated from rabbit ventral root fibers and selected geometric variables related to periaxoplasmic plaque domains. The data in $A, B, D$, and $F$ are binned values from pooled measurements of specimens stained with YOYO-1 and Y-10B (see Results). $A, \mathrm{~A}$ frequency distribution histogram of diameters of whole mounts used in the two series for analysis. $B$, A frequency histogram distribution showing the range of periaxoplasmic plaque lengths encountered in the combined series. $C$, A scatter plot of mean $\pm \mathrm{SD}$ plaque length per segment, ranked in order of increasing length. $D$, A frequency histogram of axial distances between periaxoplasmic plaques. $E$, A scatter plot of mean plaque area \pm SD per whole-mount segment, ranked in order of increasing area. $F$, A frequency distribution histogram showing the range of plaque areas per whole-mount segment. regions shown in Figure 4, $A$ and $B$, from a young $2 \mathrm{~kg}$ rabbit with those shown in Figure 4, $D$ and $E$, from an older $5 \mathrm{~kg}$ rabbit.

\section{ESI examination of periaxoplasmic plaque domains in axoplasmic whole mounts}

Generally, electron density, enhanced by heavy metal staining, and size provide the criteria for identifying ribosomes by conventional transmission electron microscopy (CTEM). When there is a search for ribosomes in cross-sectional profiles of axoplasm, however, the density of axially oriented cytoskeletal elements can give rise to structural ambiguities that contribute to uncertainty in identifying ribosomes (our unpublished observations). On the other hand, the high phosphorus content of nucleic acids lends itself to the use of ESI, a physical method in which the mapping of ribosomal phosphorus (P) signals is based on energy loss spectroscopy (Korn et al., 1983; Ottensmeyer, 1986). ESI requires an electron microscope equipped with an energy spectrometer and appropriate energy filters. When an ultrathin section $(<25 \mathrm{~nm})$ is imaged in an energy window above the $\mathrm{P}$ absorption edge (e.g., $\Delta E=155 \mathrm{eV}$ ), electrons inelastically scattered from ribosomal $\mathrm{P}$ produce a bright signal of $\sim 25 \mathrm{~nm}$ in diameter (Martin et al., 1993) in a low-contrast microscopic field. Bright phosphorus-specific signals fade when the energy window that has been selected is below the $\mathrm{P}$ absorption edge (e.g., $\Delta E=110 \mathrm{eV}$ ). ESI images of the rough endoplasmic reticulum (ER) of a cell above and below the phosphorus absorption edge may be found elsewhere (e.g., see Fig. 3; Koenig and Giuditta, 1999).

Because osmium fixation is required to stabilize myelin but is precluded for ESI examination, the available options are to use either bundles of axoplasmic whole mounts or delipidated nerve fibers (see Materials and Methods). Each of these approaches was tried for ESI on specimens prepared from rabbit ventral nerve roots. Ultrathin sections of Epon-embedded whole-mount bundles were prepared (see Materials and Methods) for examination by ESI. Figure 7 is a gallery containing examples of plaque domains, in which images were mapped above and below the phosphorus absorption edge. The whole-mount specimen images were brighter than normal both in energy loss windows above (e.g., $\Delta E=155 \mathrm{eV}$; Fig. $7 A-D)$ and below the $\mathrm{P}$ edge $\left(\Delta E=110 \mathrm{eV}\right.$; Fig. $\left.7 A^{\prime}-D^{\prime}\right)$ because of nonspecific electron scattering. This may have been caused by an increased compaction of axoplasmic mass during the preparative procedure (see Materials and Methods). Nonetheless, specific ribosome-like P signals (arrowheads) are visible above the nonspecific background brightness and fade in the energy window below the $\mathrm{P}$ absorption edge.

In longitudinal sections the plaque domains are localized in a peripheral zone of axoplasm near the surface boundary (i.e., membrane; Fig. $7 A-D$ ). In addition to $\mathrm{P}$ signals typical of ribosomes (arrowheads), there are clusters of smaller P signals (arrow) that are 

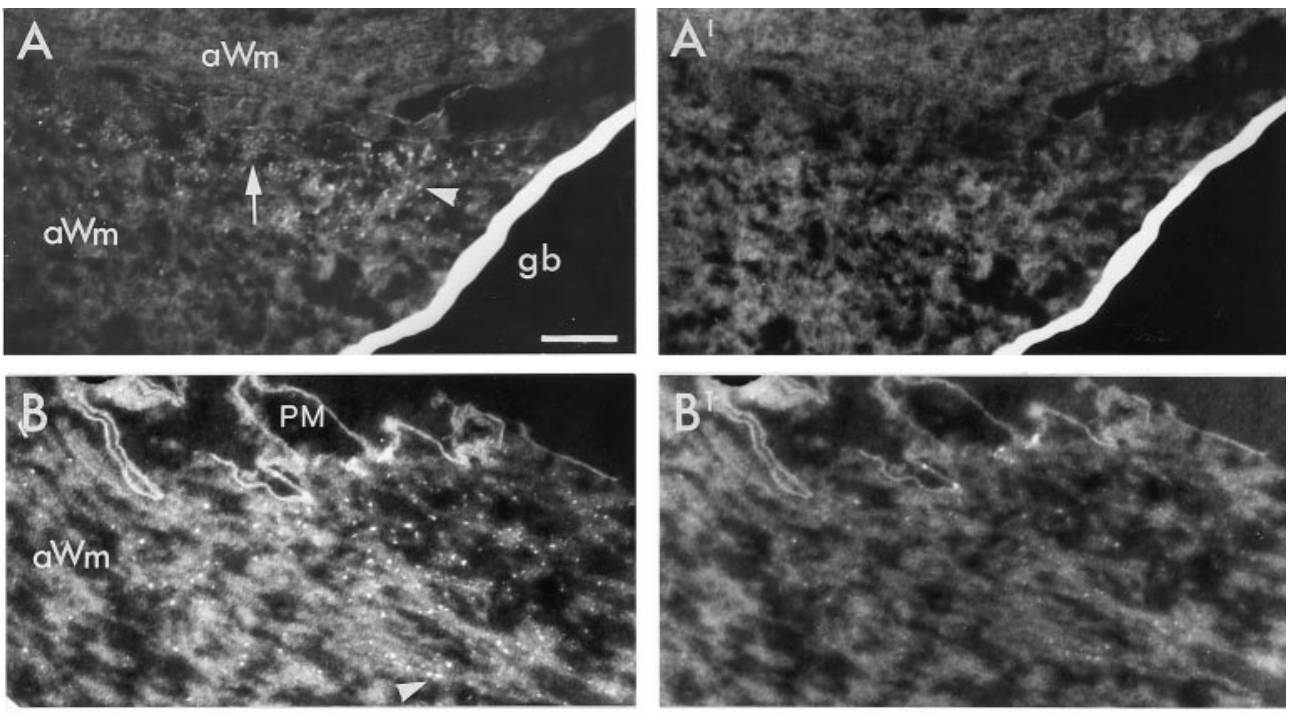

Figure 7. A gallery of selected ESI images of plaque domains in the peripheral zone of axoplasmic whole mounts isolated from rabbit ventral root fibers. $A$ contains two axoplasmic whole mounts; there is a plaque domain only in the lower whole mount. All other panels have a single whole mount exhibiting a plaque domain. $A-D$, Photomicrographs of plaque domains that were mapped in energy windows above the phosphorus absorption edge (e.g., $\Delta E=155 \mathrm{eV}$ ). $A^{\prime}-D^{\prime}$, Corresponding plaque domains mapped below the phosphorus absorption edge (e.g., $\Delta E=110 \mathrm{eV}$ ). Nonspecific electron scattering of embedded whole mounts generally increased the brightness of axoplasm in the two energy windows (see Results); nonetheless, bright P-specific signals visible in the 155 $\mathrm{eV}$ energy window faded in the $110 \mathrm{eV}$ energy window. In addition to ribosomal $\mathrm{P}$ signals (arrowheads) in plaque domains, there are also distributions of smaller, nonribosomal P signals (arrows), especially evident in $A$ and $C$. Profiles of ER cisterns in $C$, with which ribosomal $\mathrm{P}$ signals appear to be in close contact (inset), are also visible. The significance of the protuberance in $D$ is unknown. $a W m$, Axoplasmic whole mount; $g b$, grid bar; $E R$, endoplasmic reticulum; $P M$, plasma membrane. Scale bars: $A-C^{\prime}, 0.30 \mu \mathrm{m} ; C$, inset, $0.14 \mu \mathrm{m} ; D, D^{\prime}, 0.20 \mu \mathrm{m}$.
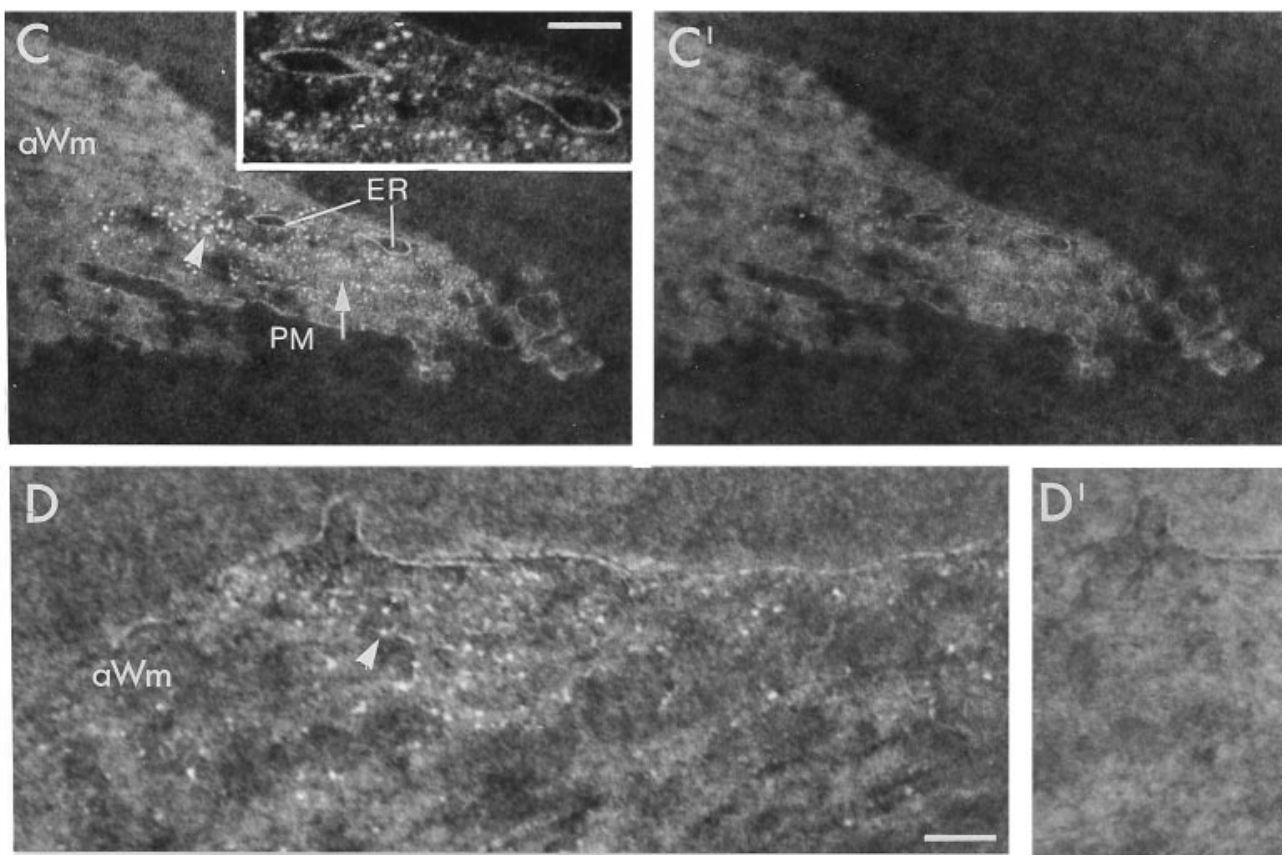

present in some instances (Fig. 7A,C), which also fade in the energy window below the $\mathrm{P}$ absorption edge (Fig. $7 A^{\prime}, C^{\prime}$ ). The smaller $\mathrm{P}$ signals could comprise partial ribosomes because the section thickness is less than that of a single ribosome; however, a cluster or delimited distribution of $\mathrm{P}$ signals of uniform size is more likely to represent a subpopulation of ribonucleoprotein particles (RNPs), which may include mRNAs (Martin et al., 1998) (also see below).

The plaque domain shown in the ultrathin section of Figure $7 C$ contains two intact membranous inclusions to which ribosome-like $\mathrm{P}$ signals appear to be attached (see Fig. $7 C$, inset). Such membrane profiles in the plaque domain suggest that they may represent ribosome-bound cisterns of an ER.

Structures that mark the plaque domain sites noted in phase microscopy (see above) were not encountered in whole-mount specimens during ESI examination of ultrathin sections. This may have been attributable to loss by disruption during preparation of the whole mounts or may have been inherent in the adventitious nature of the sampling process (however, see below).

\section{ESI examination of delipidated myelinated ventral root fibers}

Another option tested for ESI examination was to extract nerve lipids to obviate the need for osmium fixation that ordinarily is used to stabilize myelin lipids. Several organic solvents and/or procedural options that were tested proved to be unsatisfactory because of poor image contrast and quality. One procedure that offered some measure of success was the insertion of chloroform-methanol extraction and methanol washout steps after glutaraldehyde fixation, followed by rehydration before standard processing for embedding (see Materials and Methods).

Figure 8 is a low-magnification montage of a myelinated ventral root fiber shown in longitudinal profile after the delipidation procedure. Membranous components, including the axolemma, mitochondrial, and ER membranes, were not evident, and vacant spaces interspersed within the remnants of the myelin sheath made the latter appear "Swiss cheese-like." In Figure 8, the longitudinal plane of section was off axis and was so angled as to pass from the axon interior to the surface of the latter. Clusters of ribosomal $\mathrm{P}$ signals appear in three framed locations at the periphery of axoplasm, which are shown at higher magnification (Fig. 8A1-A3, arrowheads). The $\mathrm{P}$ signals in the $155 \mathrm{eV}$ energy window, which fade in the $110 \mathrm{eV}$ energy window (Fig. 8A3'), are typically ribosomal in size and appearance. Also noteworthy are highcontrast linear structures near ribosome distributions at some locations (Fig. 8A1,A2, arrows). Such structures near ribosomes are 


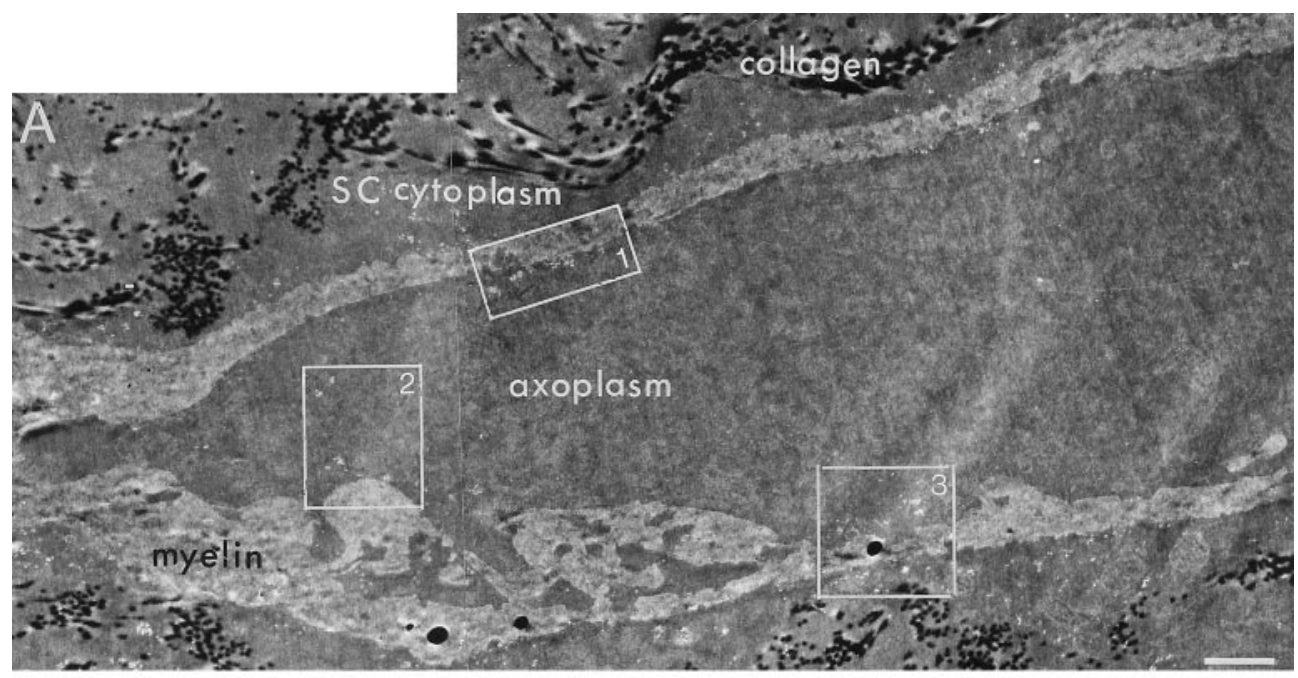

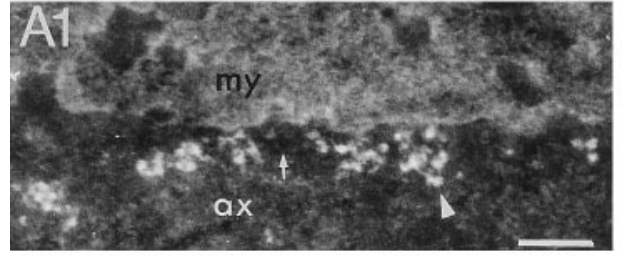
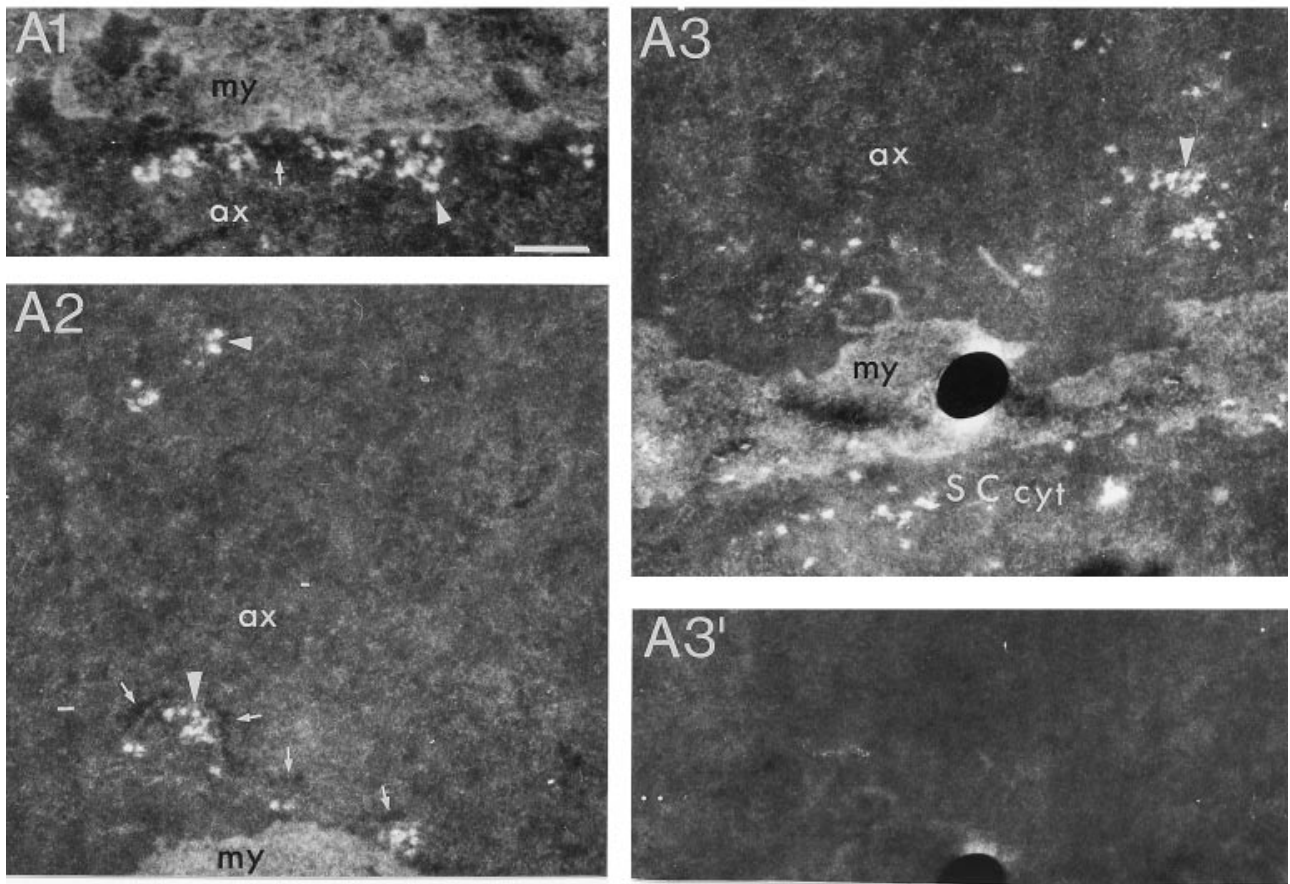

Figure 8. Ribosome distributions in a myelinated ventral nerve root fiber shown in longitudinal profile after the delipidation procedure (see Materials and Methods). Vacant spaces in the myelin sheath render it "Swiss cheese-like" in appearance. $A$, A low-magnification montage of ESI micrographs mapped in the $\mathrm{P}$ energy window (e.g., $\Delta E=155$ $\mathrm{eV})$, in which three areas with ribosome distributions are framed and identified by numbers. The plane of section was so angled as to pass from the interior of the axon (right) to the surface of the axon (left). Thus, framed area 2 is near the lateral surface of the axon. Each of the framed areas (1-3) containing ribosomes is magnified in corresponding panels $(A 1-A 3)$. Note that ribosomes (arrowheads) are distributed at the periphery (i.e., near axon surface). In addition, high-contrast linear structures (arrows) in $A 1$ and $A 2$ are located near ribosome distributions and are reminiscent of a matrix (see Results). $A 3^{\prime}$, A portion of framed area 3 shows that $\mathrm{P}$ signals in $A 3$ fade when they are mapped in an energy window below the $\mathrm{P}$ absorption edge (e.g., $\Delta E=110 \mathrm{eV}) . S C$, Schwann cell; ax, axoplasm; $c y t$, cytoplasm; $m y$, myelin. Scale bars: $A, 0.74 \mu \mathrm{m} ; A 1-A 3^{\prime}, 0.14 \mu \mathrm{m}$. reminiscent of a matrix described in Mauthner axoplasmic whole mounts (Koenig and Martin, 1996). More direct evidence of ribosome-binding properties of matrix in a plaque domain is presented below.

Portions of two ultrathin sections, cut tangential to the surface of a putative plaque domain in one block of a delipidated nerve fiber, provide additional examples of a nonribosomal RNP P signal distribution and of a ribosome-binding matrix. The inner plane of the plaque domain is shown in Figure 9. In addition to three large clusters of ribosomes in the phosphorus energy window (arrowheads) and some scattered single ribosomes, there is a cluster of smaller nonribosomal $\mathrm{P}$ signals (arrow) similar to those noted above in axoplasmic whole mounts (see Fig. 7). Such delimited distributions suggest that the plaque domain may contain one or more subdomains in which putative RNP particles may be enriched.

Figure 10 shows a portion of the outer plane of the same plaque domain in which a ribosome-binding matrix was identified also. As noted previously, a protruding structure marking the domain site was not encountered in rabbit whole mounts sampled by ESI (see above). Nonetheless, a ribosome-binding matrix became readily discernible in the plaque domain of the delipidated fiber when it was examined in an energy window above the carbon absorption edge (e.g., $\Delta E=300 \mathrm{eV}$; Fig. 10B). In this energy window the contrast of the matrix was enhanced greatly because of its much lower electron-scattering properties in comparison to those of the surrounding plastic-embedding material. The clustered and scattered single $\mathrm{P}$ signals (Fig. 10 $A$ ) appearing in an energy window above the $\mathrm{P}$ absorption edge $(\Delta E=155 \mathrm{eV}$; Fig. $10 A)$ were typical of ribosomes, and they also faded below the $\mathrm{P}$ absorption edge (data not shown). Unlike the matrix, however, ribosomes are not identified uniquely in the carbon energy window, presumably because inelastic electron scattering is not different from that of Epon.

From an inspection of ESI images in the two energy windows, the distributions of ribosomes and matrix were similar. To evaluate a potential overlap of the two, we digitized, inverted, and pseudocolored the relevant region of the $155 \mathrm{eV}$ micrograph as green; we digitized and pseudocolored the corresponding portion of the 300 eV micrograph as red. The two pseudocolored images were positioned in register, using fiduciary micrograph markings against a black background. The yellow/orange pixels (Fig. 10C) of most ribosomes, including many scattered ones, indicate common areas of overlap with matrix. It seems likely, therefore, that ribosomes and matrix do form a distinctive structural complex. 

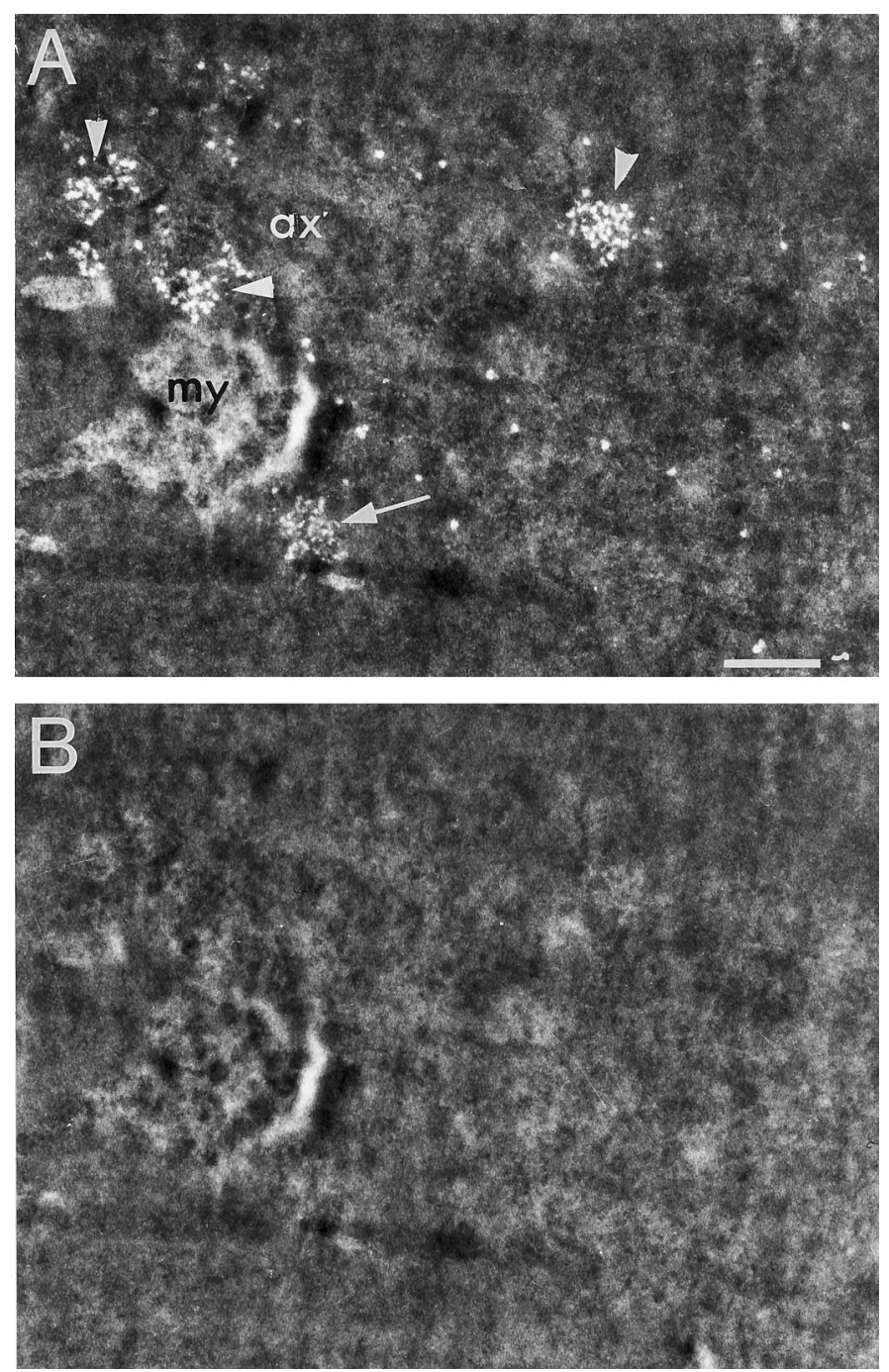

Figure 9. The inner plane of a putative plaque domain in a grazing ultrathin surface section of a delipidated ventral root nerve fiber. $A$, An ESI micrograph mapped in the $\mathrm{P}$ energy window (e.g., $\Delta E=155 \mathrm{eV}$ ) shows distributions of ribosome $\mathrm{P}$ signals (arrowheads) and a cluster of smaller, nonribosomal $\mathrm{P}$ signals (arrow). $B$, An ESI micrograph corresponding to $A$ mapped in an energy window below the $\mathrm{P}$ absorption edge (e.g., $\Delta E=110$ $\mathrm{eV}$ ), shows that the $\mathrm{P}$ signals fade. $a x$, Axoplasm; my, myelin. Scale bar, $0.26 \mu \mathrm{m}$.

\section{DISCUSSION}

The complexity in the biology of the axon has long been underestimated as a result of prevailing views about the apparent lack of an endogenous protein-synthesizing machinery, despite biochemical and molecular biological evidence to the contrary (Koenig and Giuditta, 1999; Alvarez et al., 2000). The view was predicated by the inability to verify the systematic occurrence of ribosomes with conventional electron microscopic techniques. It was not until very recently that earlier reports of rRNA in the Mauthner axon (Koenig, 1979) and in the squid giant axon (Giuditta et al., 1980) could be confirmed by documenting the systematic occurrence of ribosomes in these model axons at an ultrastructural level (Koenig and Martin, 1996; R. Martin and R. Bleher, unpublished observations). The present report extends the findings to mammalian myelinated axons.

Immunofluorescence that is based on rRNA-specific mAb Y-10B (Lerner et al., 1981) and ribosomal P antigen (Chu et al., 1991) antibodies and energy loss spectroscopic (ESI) mapping of ribosome phosphorus signals (Martin et al., 1989) provide the principal line of evidence for identifying ribosomes in spinal root axons at the light and electron microscopic levels, respectively. Unlike most cells in which ribosomes usually are distributed within the bulk volume of cytoplasm, ribosomes in axons of myelinated fibers in rabbit and rat spinal nerve roots and in the goldfish CNS (Koenig and Martin, 1996) are localized in restricted domains, distributed intermittently around the periphery of axoplasm. These restricted ribosomal domains are called periaxoplasmic plaques because of their peripheral location and an overlying structure that marks the domain sites. The structural component appears to be a matrix to which the ribosomes are attached (Koenig and Martin, 1996).

In the younger adult rabbit an average ribosomal plaque domain is $\sim 10 \mu \mathrm{m}$ long and $\sim 2 \mu \mathrm{m}$ wide (see Table 1 ); it appears from inspection to be shorter in the smaller-diameter rat axons (see Fig. 2) and perhaps longer in older rabbits (see Fig. 4). The dispersed random distribution of structures of such small dimensions near the membrane offers a challenge for detection by random sampling and sectioning techniques that are used in conventional light and electron microscopy. It is not surprising, therefore, that the systematic distribution of periaxoplasmic ribosomal domains remained undetected in ensheathed axons.

The principal findings at a light microscope level and partly at an EM level are based on the use of the axoplasmic whole-mount preparation, in which axoplasm is translated out of its myelin ensheathment for experimental observation and analysis. This preparation was used previously to analyze RNA composition (Koenig, 1965, 1979), metabolic radiolabeling of proteins (Tobias and Koenig, 1975; Frankel and Koenig, 1978; Koenig, 1991), particle transport (Koenig, 1986), and spectrin immunocytochemistry (Koenig and Repasky, 1985) of cytologically defined axoplasm. It offers an unobstructed global overview of the extended axonal compartment in which morphological and distributional features of periaxoplasmic plaques along axons can be visualized readily after appropriate staining (see Figs. 2-5). When conventional histological techniques were tested, the approach was judged to lack merit for studying plaque domains for several reasons, included among which were a low incidence of occurrence associated with the random sampling technique and the ambiguity inherent in identifying partially exposed domains with low-fluorescence intensity.

The technique of isolating axoplasm as a whole mount does have limitations, however, because the periaxoplasmic location of plaque domains makes the latter vulnerable to disruptive shear forces during translation out of the myelin sheath (see Materials and Methods, Results). Subtle variations in mechanical properties (e.g., plasticity, stiffness, compliance) of denatured axoplasm and the myelin sheath very likely play a role in the variability related to the $\mathrm{CPC}$, efficacy in recovering plaques, and structural integrity of plaque domains.

Although it remains to be demonstrated that proteins are synthesized in periaxoplasmic plaque domains, their systematic occurrence provides a ready explanation for the radiolabeling of axoplasmic proteins in mature myelinated axons sensitive to inhibitors of cytoribosomal-dependent protein synthesis (Edström, 1966; Koenig, 1967, 1991; Edström and Sjöstrand, 1969; Tobias and Koenig, 1975; Frankel and Koenig, 1978). Some of the radiolabeled polypeptides appear to be proteins identified as constituents of slow transport rate groups and include actin and tubulin in rat spinal root axons, as well as neurofilament (NF) proteins in the case of the Mauthner axon (Koenig, 1991). Such findings are also consistent with reports of mRNAs coding for NF-L in rat neurohypophyseal axons (Mohr and Richter, 1992) and NF-M in Mauthner axon (Weiner et al., 1996) as well as $\beta$-actin, $\beta$-tubulin (Kaplan et al., 1992), kinesin heavy chain (Gioio et al., 1994), enolase (Chun et al., 1995), and a calcium channel protein (Chun et al., 1997) in squid axoplasm. In situ hybridization (ISH) of $\beta$-actin mRNA in plaque domains of Mauthner axoplasmic whole mounts (J. Sotelo-Silveira, M. Crispino, and E. Koenig, unpublished observations) provides additional support for the idea that periaxoplasmic plaques are likely to be discrete centers of local translational activity in the myelinated axon. Inasmuch as they are in close proximity to the plasma membrane, there is also a potential for local regulation of protein synthesis in axons by membrane-signaling pathways. 

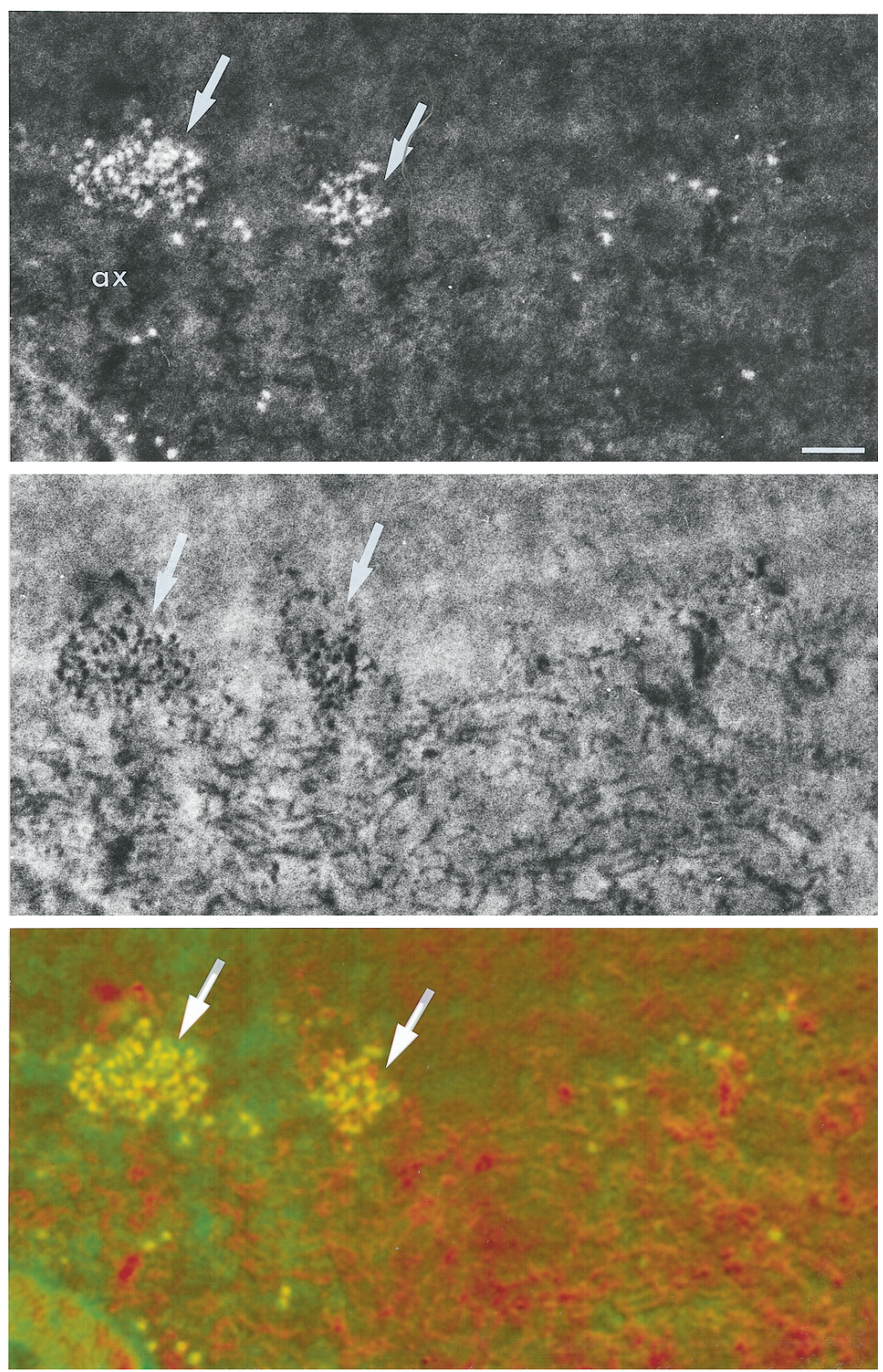

Figure 10. A portion of an outer plane of the same plaque domain shown in Figure 9, in which a ribosome-binding matrix is evident. $A$, An ESI micrograph mapped in the $\mathrm{P}$ energy window (e.g., $\Delta E=155 \mathrm{eV}$ ) shows two large ribosomal clusters (arrows) in addition to scattered single ribosomes. $B$, An ESI micrograph corresponding to the same area in $A$, mapped in an energy window above the carbon absorption edge (e.g., $\Delta E=300 \mathrm{eV}$ ). Note that the brightness of the background, because of electron scattering of the embedding plastic, enhances the contrast of the two large clusters of matrix (arrows). $C$, Digitized images of the $155 \mathrm{eV}$ ESI ribosome map, shown in $A$, were inverted and pseudocolored green, and the $300 \mathrm{eV}$ ESI matrix map, shown in $B$, was pseudocolored red and positioned in register (see Results for details). The yellow/orange pixels correspond to areas of common overlap and indicate that ribosomes and matrix may represent components of a distinctive structural complex. $a x$, Axoplasm. Scale bar, $0.15 \mu \mathrm{m}$.
If proteins are synthesized in plaque domains to satisfy requirements for local turnover of axoplasmic proteins, then the question arises as to whether the ribosome content of an axon segment is related in any way to the protein mass of that segment. Total area occupied by plaques in a whole-mount segment did not correlate with the surface area nor with the volume of the cognate segment. This may be explained in part by inconsistent recovery of intact plaques in the whole mounts that were selected for analysis or by errors inherent in the measurements themselves. It is more likely, however, that plaque area is not a valid indicator of ribosome content in a domain because ribosomes are not distributed in a two-dimensional plane. Thus, immunofluorescence intensity of Y-10B per whole-mount segment may offer a better indirect quantitative measure with which to probe the relationship between ribosome content and axoplasmic volume/mass.

In a CTEM study of fibers in dorsal nerve root of the rat, Pannese and Ledda (1991) documented an example of ribosomes attached to the surface of tubular ER in peripheral axoplasm of sensory root axons. In one plaque domain (see Fig. $7 C$ ) ribosomal $\mathrm{P}$ signals were also in close contact with putative ER cisterns. Currently, there is no evidence that ER-dependent translation or post-translational glycosylation, which has been documented in dendrites (Torre and Steward, 1996), occurs in the axon compartment. However, the calcium channel protein mRNA identified in the squid giant axon (Chun et al., 1997) is an example of at least one RNA transcript coding for an integral membrane protein in the giant squid axon. In addition, ISH evidence of 7 SL RNA and immunofluorescence of SRP 54 in axoplasmic whole mounts (I. Muslimov, M. Titmus, H. Tiedge, and E. Koenig, unpublished observations) suggests that there may be signal recognition particles (SRPs) in the axon compartment. The question of whether there is a local capability for translating membrane protein mRNA merits further investigation (see Note Added in Proof).

The occasional distinct distributions of ribosomal and small RNP-like phosphorus signals (see Figs. 7, 9) suggest a novel partitioning of the plaque domain into ribosome- and RNP-enriched subdomains. Although further work is needed to evaluate this question also, the potential significance of such a spatial segregation is unclear at present.

The protruding structure that marks the ribosomal domain in whole mounts is a singular feature that seems to characterize many plaques observed in Mauthner (Koenig and Martin, 1996), rabbit, 
and rat whole mounts (see Fig. 5). It comprises a matrix to which ribosomes appear to be bound (see Koenig and Martin, 1996) (see Fig. 10). In the squid giant axon there are ribosomal "ovoid aggregates" of $\sim 1 \mu \mathrm{m}$ in size, based on Y-10B immunofluorescence staining and ESI analysis (Martin and Bleher, unpublished data). These structural ribosomal entities are not located selectively in a periaxoplasmic zone, as in the case of myelinated axons, but they are distributed sparsely and randomly within bulk axoplasm of the giant fiber. Because ribosomal ovoid aggregates also contain a matrix and because ribosomes free of matrix were not observed (Martin and Bleher, unpublished data), they also can be considered to be "endoaxoplasmic" plaques. It would appear, therefore, that matrix and ribosomes may form a distinct structural complex in axons in which matrix could serve to anchor and/or govern the spatial distribution of ribosomes. Matrix thereby would localize translational machinery to discrete sites within the axon compartment. The notable correspondence between the shape of structural correlates and the distribution of ribosomes (see Fig. 5) supports the inference that the matrix may govern ribosome distribution in some manner.

In conclusion, the results of this report and other recent findings (see Koenig and Giuditta, 1999; Alvarez et al., 2000) indicate that traditional views of the axon as a metabolic compartment that is deficient in protein-synthesizing machinery need to be revised.

Note added in proof. The capacity to synthesize and express a functionally competent G-protein-coupled conopressin membrane receptor after microinjection of the cognate mRNA in surgically isolated axons of an identified invertebrate neuron in Lymnaea was reported recently (Spencer et al., 2000).

\section{REFERENCES}

Alvarez J, Giuditta A, Koenig E (2000) Protein synthesis in axons and terminals: significance for maintenance, plasticity, and regulation of phenotype. With a critique of slow transport theory. Prog Neurobiol 62:1-62.

Barker L (1899) The nervous system, p 307. New York: Appleton.

Black M, Lasek RJ (1980) Slow components of axonal transport: two cytoskeletal networks. J Cell Biol 86:616-623.

Chu J-L, Brot N, Weissbach H, Elkon K (1991) Lupus antiribosomal P antisera contain antibodies to a small fragment of $28 \mathrm{~S}$ rRNA located in the proposed ribosomal GTPase center. J Exp Med 507-514.

Chun JT, Gioio AE, Crispino M, Giuditta A, Kaplan BB (1995) Characterization of squid enolase mRNA: sequence analysis, tissue distribution, and axonal localization. Neurochem Res 20:923-930.

Chun JT, Gioio AE, Crispino M, Eyman M, Giuditta A, Kaplan BB (1997) Molecular cloning and characterization of a novel mRNA present in the squid giant axon. J Neurosci Res 49:144-153.

Edström A (1966) Amino acid incorporation in isolated Mauthner nerve fibre of goldfish. J Neurochem 13:315-321.

Edström A, Sjöstrand J (1969) Protein synthesis in isolated Mauthner nerve fibre components. J Neurochem 16:67-81.

Frankel RD, Koenig E (1978) Identification of locally synthesized proteins in proximal stump axons of neurotomized hypoglossal nerve. Brain Res 141:67-76.

Gilbert DS, Newby BJ, Anderton BH (1975) Neurofilament disguise, destruction and disciplines. Nature 256:586-589.

Gioio AE, Chun JT, Crispino M, Capano CP, Giuditta A, Kaplan BB (1994) Kinesin mRNA is present in the squid giant axon. J Neurochem 63:13-18.

Giuditta A (1980) Origin of axoplasmic protein in the squid giant axon. Riv Biol (Italy) 73:35-49.

Giuditta A, Cupello A, Lazzarini G (1980) Ribosomal RNA in the axoplasm of the squid giant axon. J Neurochem 34:1757-1760.

Grafstein B, Foreman DS (1980) Intracellular transport in neurons. Physiol Rev 60:1167-1283.

Hirokawa N (1991) Molecular architecture and dynamics of the neuronal cytoskeleton. In: The neuronal cytoskeleton (Burgoyne RD, ed), pp 5-74. New York: Wiley.

Kaplan BB, Gioio AE, Perrone Capano C, Crispino M, Giuditta A (1992) $\beta$-Actin and $\beta$-tubulin are components of a heterogeneous mRNA population present in the squid giant axon. Mol Cell Neurosci 3:133-144.
Koenig E (1965) Synthetic mechanisms in the axon. II. RNA in myelinfree axons of the cat. J Neurochem 12:357-361.

Koenig E (1967) Synthetic mechanisms in the axon. IV. In vitro incorporation into axonal protein and RNA. J Neurochem 14:437-446.

Koenig E (1979) Ribosomal RNA in Mauthner axon: implications for a protein synthesizing machinery in the myelinated axon. Brain Res 175:95-107.

Koenig E (1984) Local synthesis of axonal protein. In: Handbook of neurochemistry, Vol 7 (Lajtha A, ed), pp 315-340. New York: Plenum.

Koenig E (1986) Isolation of native Mauthner cell axoplasm and an analysis of organelle movement in non-aqueous and aqueous media. Brain Res 398:288-297.

Koenig E (1991) Evaluation of local synthesis of axonal proteins in the goldfish Mauthner cell axon and axons of dorsal and ventral roots of the rat in vitro. Mol Cell Neurosci 2:384-394.

Koenig E, Giuditta A (1999) Protein synthesizing machinery in the axon compartment. Neuroscience 89:5-15.

Koenig E, Martin R (1996) Cortical plaque-like structures identify ribosome-containing domains in the Mauthner axon. $\mathrm{J}$ Neurosci 16:1400-1411.

Koenig E, Repasky E (1985) A regional analysis of $\alpha$-spectrin in isolated Mauthner neuron and in isolated axons of the goldfish and rabbit. J Neurosci 5:705-714.

Korn AP, Spitnik-Elson P, Elson D, Ottensmeyer FP (1983) Specific visualization of ribosomal RNA in the intact ribosome by electron spectroscopic imaging. Eur J Cell Biol 31:334-340.

Lasek RJ, Hoffman PN (1976) The neuronal cytoskeleton, axonal transport, and axonal growth. In: Cell motility: microtubules and related proteins (Goldman R, Pollard T, Rosenbaum J, eds), pp 1021-1049. New York: Cold Spring Harbor.

Lerner EA, Lerner MR, Janeway Jr CA, Steitz JA (1981) Monoclonal antibodies to nucleic acid-containing cellular constituents: probes for molecular biology and autoimmune disease. Proc Natl Acad Sci USA 78:2737-2741.

Martin R, Fritz W, Giuditta A (1989) Visualization of polyribosomes in the postsynaptic area of the squid giant synapse by electron spectroscopic imaging. J Neurocytol 418:11-18.

Martin R, Door R, Breitig D (1993) High resolution imaging of protein phosphorylation in the squid giant axon and synapse. J Histochem Cytochem 41:1133-1139.

Martin R, Vaida B, Bleher R, Crispino M, Giuditta A (1998) Protein synthesizing units in presynaptic and postsynaptic domains of squid neurons. J Cell Sci 111:3157-3166.

Mohr E, Richter D (1992) Complexity of mRNAs in the axonal compartment of peptidergic neurons in the rat. Eur J Neurosci 4:870-876.

Nixon RA (1980) Protein degradation in the mouse visual system. I. Degradation of axonally transported and retinal proteins. Brain Res 200:69-83.

Nixon RA, Logvinenko KB (1986) Multiple fates of newly synthesized neurofilament proteins: evidence for a stationary neurofilament network distributed nonuniformly along axons of retinal ganglion cell neurons. J Cell Biol 102:647-659.

Ottensmeyer FP (1986) Elemental mapping by energy filtration: advantages, limitations, and compromises. Ann NY Acad Sci 483:339-353.

Palay SL, Palade GE (1955) The fine structure of neurons. J Biophys Biochem Cytol 1:69-88.

Pannese E, Ledda M (1991) Ribosomes in myelinated axons of the rabbit spinal ganglion neurons. J Submicrosc Cytol Pathol 23:33-38.

Peters A, Palay SL, Webster HF (1970) The fine structure of the nervous system. New York: Harper and Row.

Sotelo JR, Kun A, Benech JC, Giuditta A, Morillas J, Benech CR (1999) Ribosomes and polyribosomes are present in the squid giant axon: an immunocytochemical study. Neuroscience 90:705-715.

Spencer GE, Syed NI, van Kesteren E, Lukowiak K, Geraets WPM, van Minnen J (2000) Synthesis and functional integration of a neurotransmitter receptor in isolated invertebrate axons. J Neurobiol 44:72-81.

Tobias GS, Koenig E (1975) Axonal protein synthesizing activity during the early outgrowth period following neurotomy. Exp Neurol 49:221-234.

Torre ER, Steward O (1996) Protein synthesis within dendrites: glycosylation of newly synthesized proteins in dendrites of hippocampal neurons in culture. J Neurosci 16:5967-5978.

Weiner OD, Zorn AM, Krieg PA, Bittner GD (1996) Medium-weight neurofilament mRNA in goldfish Mauthner axoplasm. Neurosci Lett 213:83-86.

Zelená J (1972) Ribosomes in myelinated axons of dorsal root ganglia. Z Zellforsch Mikrosk Anat 124:217-229. 